\title{
Overview of the modified magnetoelastic method applicability
}

\author{
Tomáš Klier ${ }^{1}$, Tomáš Míčka ${ }^{1}$, Michal Polák ${ }^{2}$, Milan Hedbávný ${ }^{3}$ \\ 1 Pontex, Bezová 1658, 147 14, Prague 4, Czech Republic \\ ${ }^{2}$ Faculty of Civil Engineering, Czech Technical University in Prague, Thákurova 7, 166 29, Prague 6, Czech Republic \\ ${ }^{3}$ Freyssinet CZ, Zápy 267, 250 01, Brandýs nad Labem, Czech Republic
}

\begin{abstract}
A requirement of axial force determination in important structural elements of a building or engineering structure during its construction or operational state is very frequent in technical practice. In civil engineering practice, five experimental techniques are usually used for evaluation of axial tensile forces in these elements. Each of them has its advantages and disadvantages. One of these methods is the magnetoelastic method, that can be used, for example, on engineering structures for experimental determination of the axial forces in prestressed structural elements made of ferromagnetic materials, e.g., prestressed bars, wires and strands. The article presents general principles of the magnetoelastic method, the magnetoelastic sensor layout and actual information and knowledge about practical application of the new approach based on the magnetoelastic principle on prestressed concrete structures. Subsequently, recent results of the experimental verification and the in-situ application of the method are described in the text. The described experimental approach is usable not only for newly built structures but in particular for existing ones. Furthermore, this approach is the only one effectively usable experimental method for determination of the prestressed force on existing prestressed concrete structures in many cases in the technical practice.
\end{abstract}

\section{Section: RESEARCH PAPER}

Keywords: Tensile force; magnetoelastic method; prestressed strand; prestressed cable; sensor

Citation: Tomáš Klier, Tomáš Mícka, Michal Polák, Milan Hedbávný, Overview of the modified magnetoelastic method applicability, Acta IMEKO, vol. 10, no. 3, article 23, September 2021, identifier: IMEKO-ACTA-10 (2021)-03-23

Section Editor: Lorenzo Ciani, University of Florence, Italy

Received February 9, 2021; In final form July 30, 2021; Published September 2021

Copyright: This is an open-access article distributed under the terms of the Creative Commons Attribution 3.0 License, which permits unrestricted use, distribution, and reproduction in any medium, provided the original author and source are credited.

Funding: This work was supported by Ministry of Industry and Trade of the Czech Republic.

Corresponding author: Tomáš Klier, e-mail: tkl@pontex.cz

\section{INTRODUCTION}

Five experimental techniques are usually applied in civil engineering practice for evaluation and verification of actual values of axial tensile forces in important structural elements on building constructions.

If the total value of the tensile force in the investigated structural elements has to be determined, the two of these methods (namely, the direct measurement of the force by a preinstalled load cell and the approach based on a strain measurement with strain gauges) can be applied only for an experiment in which the applied sensors were installed before the investigated structural elements were activated.

Compared to that, the next three methods (namely, the vibration frequency method [1], [2], the force determination in a flexible structural element based on the relation between the transverse force and the caused transverse displacement [3]-[5] and the magnetoelastic method [6]-[24] can be used during newly started experiments on existing structures that have already been in service for some time. The basic advantage of these three methods is that the investigated structural elements remain activated all the time (namely, in the period of the structure service, the experiment preparation, the experiment realization and also after the experiment completion).

However, the applicability of the method using the relation between the transverse force and the element transverse displacement is significantly limited in practice. It can be effectively applied only for the flexible elements with a relatively small cross section, as are strands with diameter smaller than approximately $25 \mathrm{~mm}$, and with a relatively long free length, because the measurable transverse displacement increases considerably the observed tensile force in the substantially short 
investigated elements even in the order of tens of percent in some special cases.

Similarly, the vibration frequency method is suitable for application on structural elements with a relatively long free vibrating length and with a relatively low bending stiffness. If this method is used on the relatively short one, the uncertainty of the specified tensile force is significantly influenced by the element bending stiffness and by the element boundary conditions, especially if they are complicated and vague. In this case, the results obtained by the vibration frequency method can be improved not only by using measured natural frequencies of the element but also by using measured mode shapes in the process of results evaluation [1], [2]. However, the time necessary for realization of such experiment is significantly longer compared to the similar one, when only the natural frequencies of the elements are evaluated, especially in the situation, if the tested elements are difficult to access for the installation of vibration sensors, as are, for example, cable stays on cable-stayed bridges. Then the time required for the experiment can be more than ten times longer.

The next advantages and disadvantages of all five above mentioned experimental techniques are discussed in more detail in the reference [11].

The selection of the most suitable experimental method for a particular practical application depends on specific element parameters and specific conditions in which the experiment is going to be performed.

According to the authors opinion, the modified magnetoelastic method is the only one that can be applied effectively and expediently for evaluation of the prestressed force on existing structures made from the prestressed concrete when the prestressed reinforcement is embedded inside the concrete.

\section{RELATED RESULTS IN THE LITERATURE}

In civil engineering practice, the utilization of the magnetoelastic method for experimental evaluation of axial tensile forces in structural elements started about thirty-five years ago. The original inventors developed gradually the method theory, the magnetoelastic sensors (hereinafter the ME sensors) and their practical utilization. They published their obtained knowledge regularly, see [12]-[21] for example.

The ME sensors and their appropriate equipment that have been standardly used in civil engineering practice in the recent past and at present [12]-[24] evaluate measured prestressed forces in a relatively simple way. These standard ME sensors are composed from two basic parts only, from the primary and secondary coil. This is the minimal possible configuration of the ME sensor, as it is described below.

The basic advantages of the application of the ME sensor in its standard configuration are that the tensile force in the structural element is evaluated contactless, the observed element is not locally deformed and its anti-corrosive layer is not abraded, the sensor body is robust, long lasting and resistant to accidental mechanical damage. It is possible to evaluate the instantaneous magnitude of the tensile force with high accuracy. However, the important requirement for high accuracy of the obtained results is the sensitivity assessment of each particular standard ME sensor in concrete conditions its practical application using an independent force sensor. In the case of the prestressed reinforcement (namely the strand or the cable), the force sensor is used as a part of a hydraulic jack and therefore it is necessary to install the ME sensor before the activation of the observed prestressed reinforcement.

An additional installation of the standard ME sensor on the activated prestressed reinforcement is, of course, technologically possible. However, it is time consuming and the sensor sensitivity assessment cannot be realized in the concrete conditions of the magnetic surroundings of the location where the ME sensor is installed.

The modified magnetoelastic method, its physical principle, the fully equipped ME sensor, an experiment on a real structure and its result evaluation are described in more details in reference [9]. Other supplementary information about the method, its practical applications and about a removable ME sensor can be found in references [6]-[8] and [10],[11].

\section{DESCRIPTION OF THE METHOD}

The method is based on an experimental estimation of the magnetic response of the tensile stressed structural element on an external magnetic field. The magnetic field intensity $\mathrm{H}$ and the magnetic flux density $\mathrm{B}$ are ones of the basic physical quantities describing the magnetic field arrangement. The relation between $\mathrm{B}$ and $\mathrm{H}$ in the form of the so-called hysteresis loop is given by the kind of material exposed to the effects of the magnetic field, its properties and its current conditions (e.g., its tensile stress and temperature).

For the purposes of applications of the modified magnetoelastic method, differently arranged ME sensors are used depending on the specific experiment and its concrete conditions.

A diagram of a fully equipped cylindrical ME sensor is shown in Figure 1 that was adopted from reference [9]. Fundamental components of this $\mathrm{ME}$ sensor variant are a controlled magnetic field source (for example, the primary coil that is drawn in Figure 1 ), a sensor of magnetic field intensity " $\mathrm{H}$ " in a measured cross section (the system of Hall's sensors and/or the secondary coil 2), a sensor of magnetic flux that is closely related to the magnetic induction "B" in the measured section of the strand (the secondary coil 1) and the ME sensor protection against magnetic influences from its surroundings (the steel shield). The function and principle of Hall's sensors were explained in more detail in the reference [10].

The fully featured ME sensor offers the greatest possibilities to increase accuracy and reduce uncertainties in evaluating of the tension force in the observed prestressed element. On the other side, the fully equipped ME sensor is spatially larger and that may restrict its applicability in some practical cases and it is also more

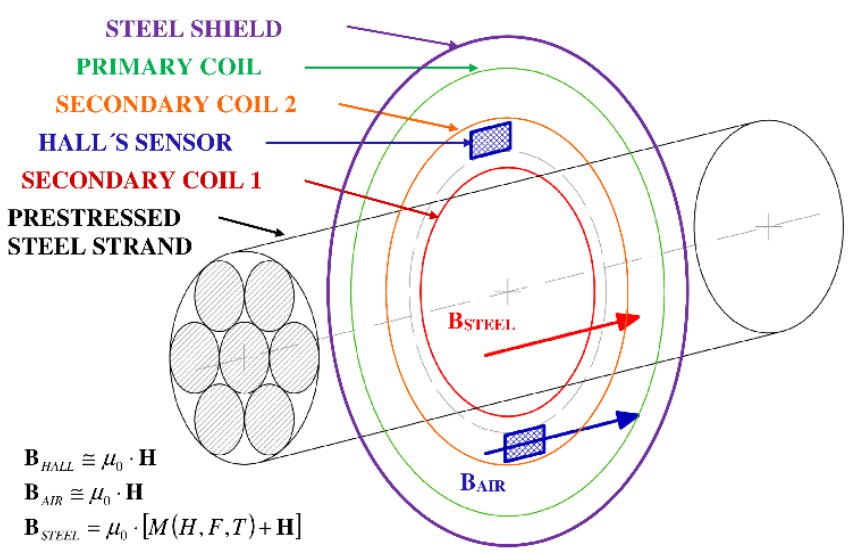

Figure 1. Diagram of a fully equipped ME sensor published also in [9] 
complicated. This complexity affects its longer production time, higher requirements for its application in civil engineering practice and as well as higher requirements on the used measuring system and its equipment.

The above-described standard ME sensors (see chapter 2) represent the minimalist variant of the ME sensor that consists of a primary coil and a secondary coil 1 only. The intensity of the magnetic field " $\mathrm{H}$ " is determined, in this case, indirectly from a completely different physical quantity, which is the electric current flowing through the primary coil. However, there is a risk that results from this approach with application of the minimalist variant of the ME sensor can be affected. Any change in the magnetic surroundings in the sensor vicinity (e.g., a removal of a massive steel falsework after concrete hardening) causes completely "silently" substantial or even severe changes in sensor parameters.

The sensor's steel shielding reduces the impact of this effect on the obtained results. The quality of the ME sensor shielding influences the level of this reduction, however, it is never one hundred percent. For example, the application of the minimum $\mathrm{ME}$ sensor configuration on a prestressed prefabricated concrete structure reinforced by steel-fibre is completely unusable.

\section{EXPERIMENTAL ANALYSIS OF EVALUATING CURVES}

For the purpose of the modified magnetoelastic method, magnetic behaviour of the standard prestressed elements used both in the past and today is appropriate and necessary to know.

In November 2019, a laboratory experiment concentrated on the systematic study of variations in the magnetic behaviour of two selected standard prestressed elements (namely, the patented wire P7, that was applied in the past, and the prestressed strand Lp15.7 currently used by the Freyssinet company with righthanded threading). It was investigated the magnetic behaviour of both elements especially in dependence on its immediate temperature and its rate of the mechanical stress. This experiment was realized in the experimental centre of the Klokner institute (the research institute at the Czech Technical University in Prague). Results of similar experiments realized for different standard prestressed elements are described in [10] (namely, for the patented wires P4.5, unknown prestressed strand Lp15.7 with left-handed threading, the prestressed bars

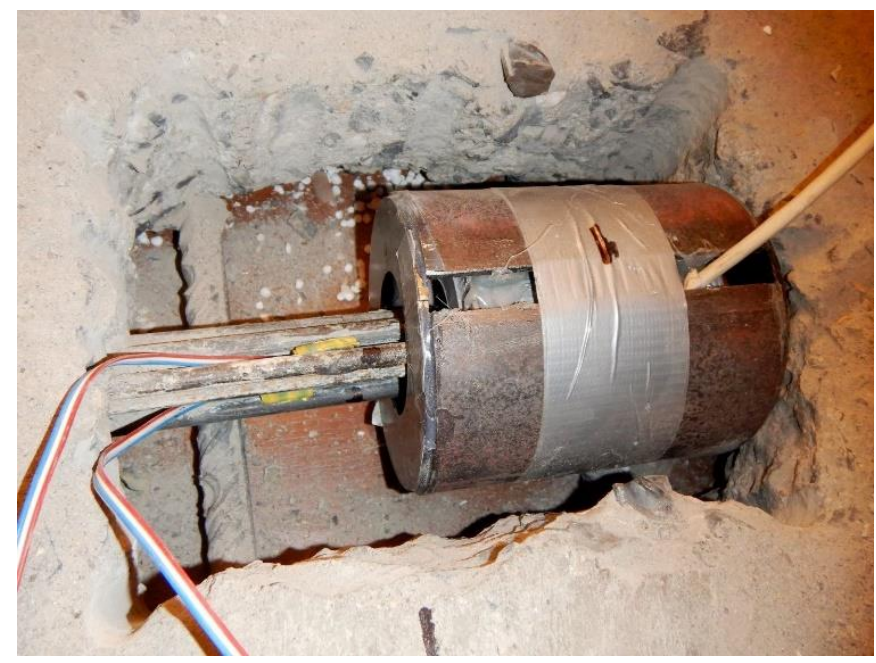

Figure 2. The fully equipped ME sensor installed on the prestressed cable in the inspected bridge
15/17 made by Dywidag company) and in [8] (namely, for the full locked cable PV 150 and the Mukusol threadbar 15FS 0000).

The ideal condition for precise evaluation of some particular experiment realized on an existing prestressed concrete structure is, when the specific evaluating curve is available. This specific curve can be obtained only by the magnetoelastic analysis of the specific prestressed element, which was removed directly from the investigated structure. The specimen should be minimally $1.2 \mathrm{~m}$ long and its extraction is generally very difficult and laborious. Moreover, the load bearing system of the observed structure is partially weakened. Also, the realization and evaluation of the experimental analysis necessary for determination of the evaluating curve is significantly timeconsuming.

Alternatively, it is possible to use the general evaluating curves that are available in the material library gradually compiled by the authors, some examples are shown in Figure 7. The results of the chemical composition and the microscopic structure analysis realized for the material of the investigated prestressed reinforcement can be used for the selection of the appropriate general evaluating curve from the material library.

The substantially shorter test specimen is needed for this purpose. Usually only one wire $10 \mathrm{~cm}$ long is sufficient enough even if it is removed from the strand. The structure weakening is generally acceptable in this scale and the material sample can be removed from the opening used for installation of the ME sensor without the additional partial damage of the structure.

\subsection{The experiment realized on the previously used patent wire P7}

The patent wire $\mathrm{P} 7$ used during the experiment described in this chapter was removed from the chamber of the existing prestressed concrete bridge in Prague that was put into operation in the year 1974 .

In the course of the bridge inspection in the year 2019, the fully equipped ME sensors were installed on two selected prestressed cables assembled from twelve patent wires P7 (see Figure 2). The opening created for the purpose of the ME sensor installation was consequently filled in by the special grout (see Figure 3). The installed ME sensors are intended for long-term monitoring of the prestressed forces in the selected cables.

The basic reason for realization of the experiment described in this chapter was to determine the accurate parameters of magnetic behaviour of the prestressed reinforcement used in the

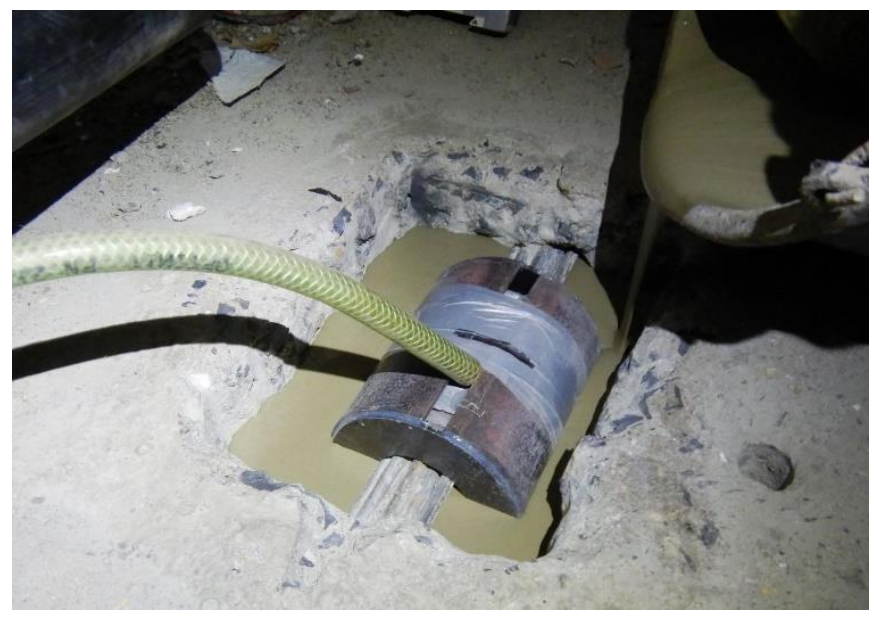

Figure 3. The fully equipped ME sensor installed on the prestressed cable, consequent filling of the created opening by the special grout 

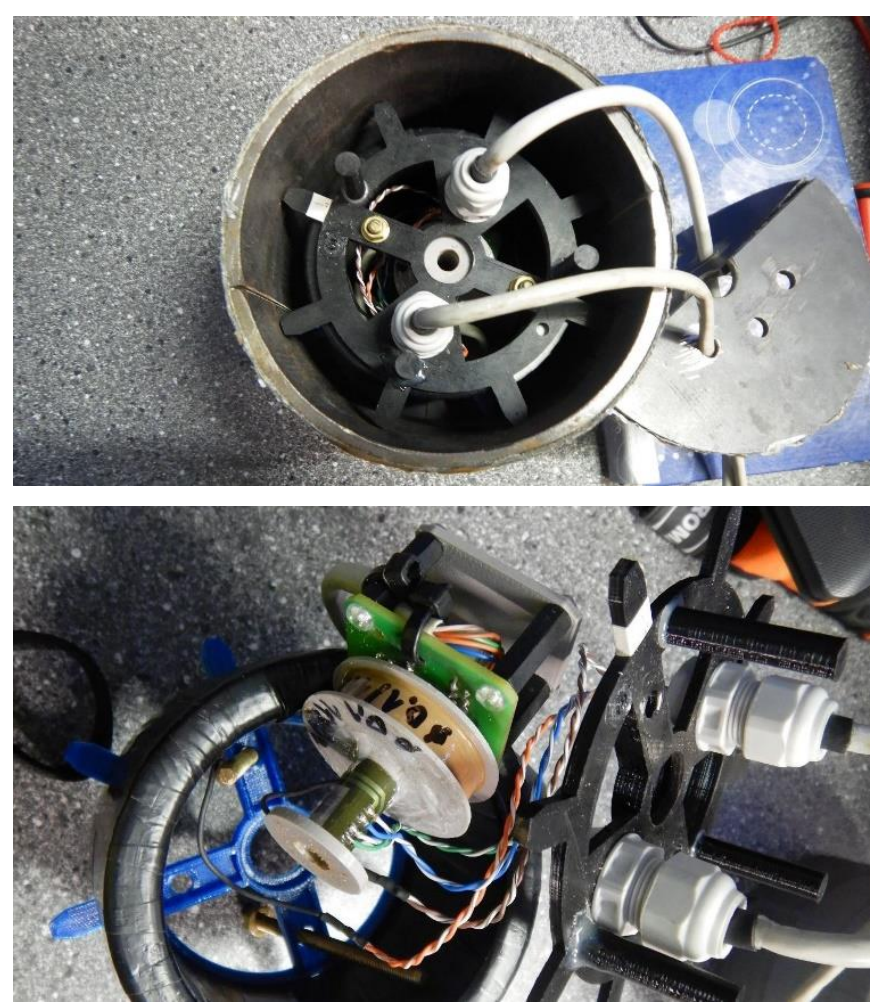

Figure 4. The fully equipped laboratory ME sensor intended for the experiment on the patent wire $\mathrm{P} 7$, the assembled sensor inside the steel shield (above) and view on its disassembled basic parts (below)

inspected bridge for more precise evaluation of the results. The fully equipped laboratory ME sensor used in the course of the experiment is shown in Figure 4.

In the course of the experiment, the investigated patent wire P7 was placed in a climatic chamber (see Figure 5) and loaded in a steel tensile testing machine. The magnetic properties of the studied wire were investigated for two temperature levels of the wire surface, namely around $0^{\circ} \mathrm{C}$ and around $+25{ }^{\circ} \mathrm{C}$. The studied patent wire P7 was loaded in five force steps for each temperature level according to its design resistance, namely, $10 \mathrm{kN}, 20 \mathrm{kN}, 30 \mathrm{kN}, 40 \mathrm{kN}$ and $47 \mathrm{kN}$. It is roughly about $20 \%$, $40 \%, 60 \%, 80 \%$ and $100 \%$ of the design strength.

The temperature of the observed wire cross-section was evaluated as a linear interpolation between two measured temperature values. The first one was observed on the element surface in the close vicinity of the ME sensor and the second one was the temperature of the air measured inside the ME sensor.

The specific hysteresis loop was measured and evaluated ten times for each particular temperature level and force step. The hysteresis loop, in general, characterizes the relation between magnetic flux density $\mathrm{B}$ and the magnetic field intensity $\mathrm{H}$ and it changes its shape depending on the actual force magnitude in the investigated prestressed element and also on its temperature. However, it is not effective and also necessary to evaluate the measured hysteresis loops in their whole range.

The dimensionless parameter $P$ is used to convert a complex measured shape of the hysteresis loop, that depends on the actual force magnitude, to one simple numeric value. And this parameter is standardly evaluated for the purpose of a practical application of the modified magnetoelastic method. Some examples are published in [6]-[11].

The particular parameter $P$ is described as a fraction. The numerator is the most important value for evaluation of the parameter $P$ and it describes the level of the magnetic field intensity " $\mathrm{H}$ " in the main node point. The numerator value of the parameter $P$ indicates the preference of the portion of the hysteresis loop close to the remanence (the intersection with the vertical axis in the $\mathrm{B}-\mathrm{H}$ curve). On the contrary, its denominator value prefers the loop portion near to the saturation. The more exact definition of the parameter $P$ is an industrial secret.

In the course of analysis of the experiment results, several parameters $P$ with different definitions were evaluated, for example parameters $P 10 / 45, P 15 / 45$ or $P 20 / 45$. The dimensionless parameter $P 15 / 45$ was finally chosen as the most suitable one for further analysis of the particular examined patent wire P7 and eventually for another similar one because of its maximal sensitivity to the prestressing force and its minimal disturbance by negative influences.

The resultant regression fitting curve using polynomial regressions was calculated for the investigated patent wire $\mathrm{P} 7$ and the chosen resultant parameter $P 15 / 45$. The temperature effect on the parameter $P$ was considered as linear and the force effect was considered as $3^{\text {rd }}$ degree polynomial. Calculated curve is one of several ones, which is drawn as curve "D7" in Figure 7. The differences between the theoretical fitting curve and the used input experimental results are small. The maximal deviation between them is $1.6 \%$ of the design strength of the investigated patent wire and the standard deviation of all particular results is $0.6 \%$ of the design strength.

\subsection{The experiment realized on the currently used prestressed strand Lp 15.7}

The prestressed strand Lp 15.7, that was the subject of the experiment described in this chapter, is standardly used by the Freyssinet company at the present time.

The arrangement of the experiment, its procedure and results evaluation were similar to those described in the previous chapter.

In the course of the experiment, the investigated prestressed strand was placed in the climatic chamber again (see Figure 5)
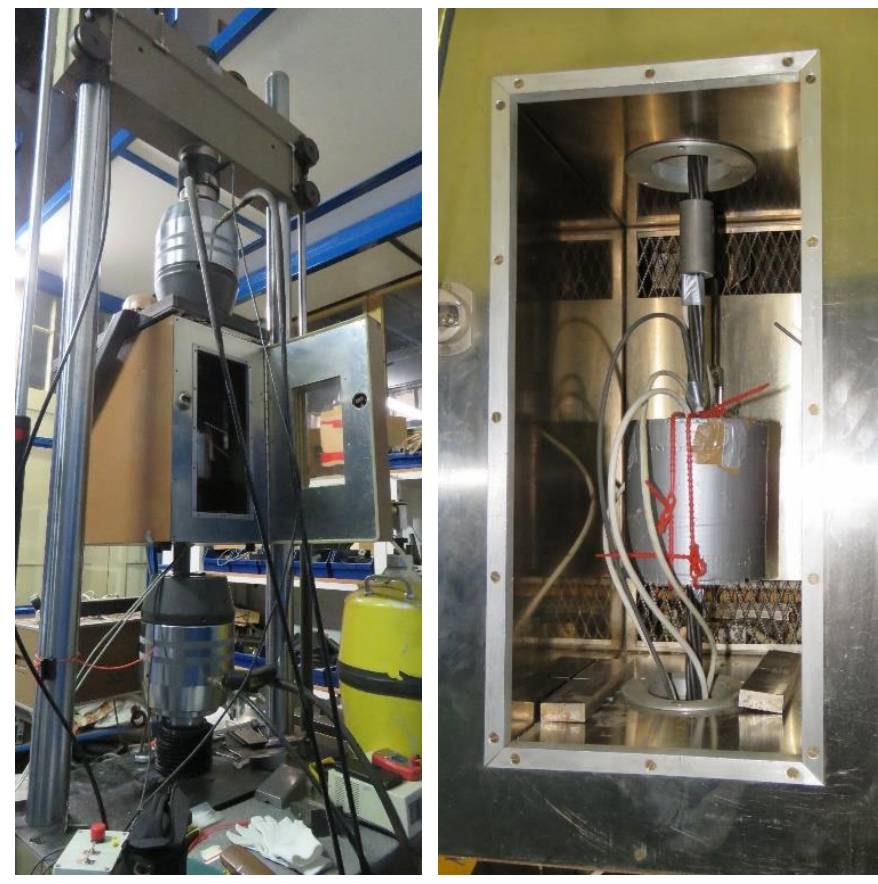

Figure 5. The exterior view on the climatic chamber and the steel tensile testing machine (on the left) and on the laboratory ME sensor installed on the prestressed strand Lp15.7 inside the chamber (on the right) 


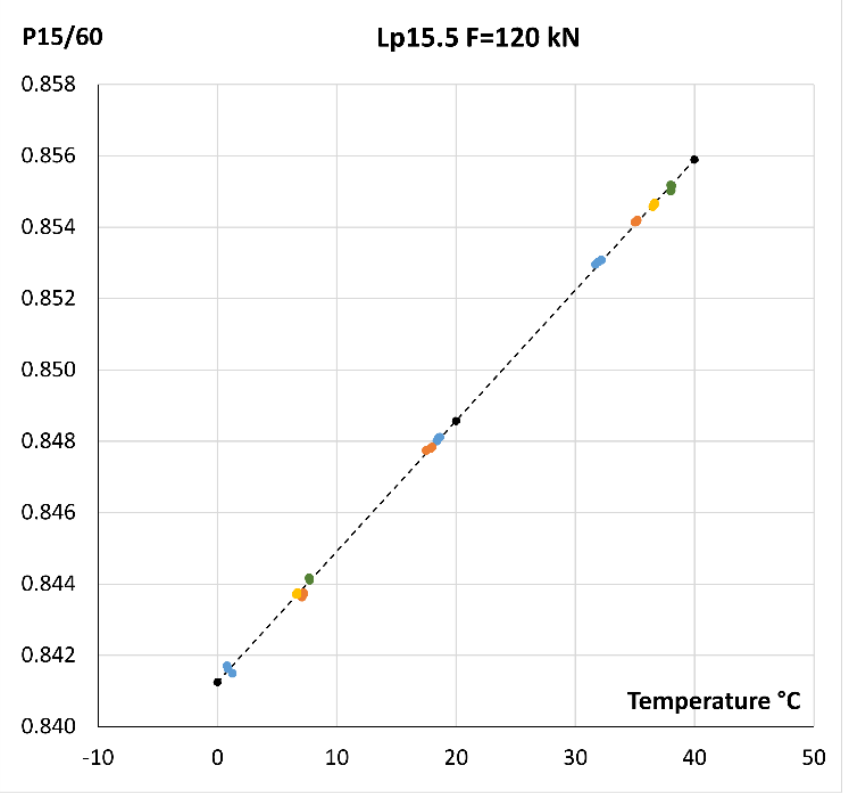

Figure 6. The prestressed strand Lp 15.7 - the relation between the temperature of the observed strand and the chosen resultant dimensionless parameter $\mathrm{P}$ 15/60 on the specific force step (120 kN)

and loaded in the steel tensile testing machine. The magnetic properties of the studied strand were investigated for three temperature levels of the strand surface namely, around $0{ }^{\circ} \mathrm{C}$, $+20{ }^{\circ} \mathrm{C}$ and $+35^{\circ} \mathrm{C}$. The strand was loaded in five force steps for each temperature level, namely, $40 \mathrm{kN}, 80 \mathrm{kN}, 120 \mathrm{kN}, 160$ $\mathrm{kN}$ and $200 \mathrm{kN}$. It is roughly about $20 \%, 40 \%, 60 \%, 80 \%$ and $100 \%$ of the strand design resistance. The temperature of the observed strand was evaluated in the same way as for the patent wire P7 in chapter 4.1.

The specific hysteresis loop was measured and determined again multiple times for each particular temperature level and force step as can be seen, for example, from Figure 6 .

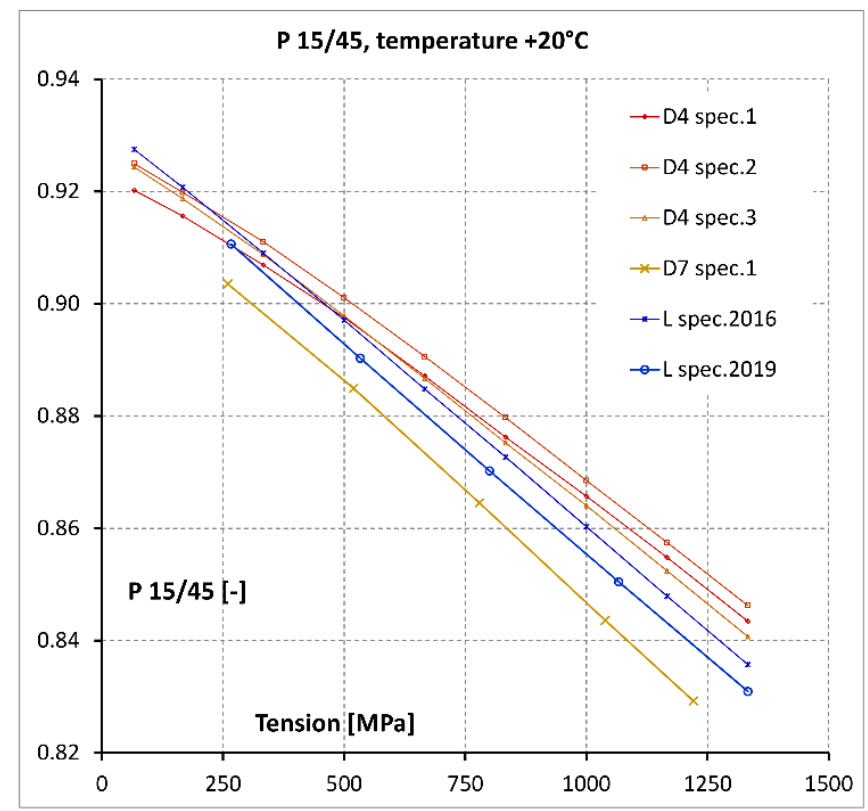

Figure 7. The comparison of the relations between the stress in the six observed prestressed elements (three patent wires P4.5, one patent wire P7 and two strands Lp15.7 / $1860 \mathrm{MPa}$ ) and the chosen resultant dimensionless parameter $P 15 / 45$ for the prestressed element temperature $20^{\circ} \mathrm{C}$
For the observed strand Lp 15.7, the example of evaluated dependence of the chosen resultant parameter $P 15 / 60$ on the strand temperature (prestressed force is constant $120 \mathrm{kN}$ ) is shown in Figure 6.

The resultant regressive fitting curves for the several chosen parameters $P$ were also calculated using the same methods of mathematical analysis and statistics analogous with chapter 4.1. The differences between the theoretical fitting curves and the used input experimental results are even smaller than for the wire P7. The maximal deviation between them is $1.4 \%$ of the design resistance of the observed prestressed strand and the standard deviation of all particular results is $0.2 \%$ of the design strength.

The example of calculated regression fitting curve that expresses the dependence of the particular chosen parameter $P$ $15 / 45$ on the stress in the observed strand at the strand temperature $20^{\circ} \mathrm{C}$ is shown in Figure 7 where the relevant curve is labelled "L 2019".

\section{PRACTICAL APPLICATION OF THE METHOD ON DIFFERENT PRESTRESSED ELEMENTS}

In this chapter, a brief summary of typical utilizations of the modified magnetoelastic method is described on some practical applications.

\subsection{The experiment realized on the prestressed cables composed of twelve strands on an existing bridge}

The actual tensile forces in prestressed cables on an existing prestressed concrete bridge were investigated during this experiment.

In general, the specific position for installation of the ME sensor on the load bearing structure of an existing bridge made from prestressed concrete is usually chosen based on three basic reasons. Firstly, the created opening in concrete (see Figure 2, Figure 3 or Figure 8 for example) does not weaken substantially the bridge load bearing structure. Secondly, the substantial degradation of the concrete or some prestressed reinforcement is supposed in the selected location, for example where water with chlorides penetrates into the structure. Thirdly, the position is chosen based on an interest of the structural designer.

The investigated bridge is about thirty years old and its prestressed system is composed of the longitudinal prestressing cables. The typical prestressed cable on this bridge is the post-

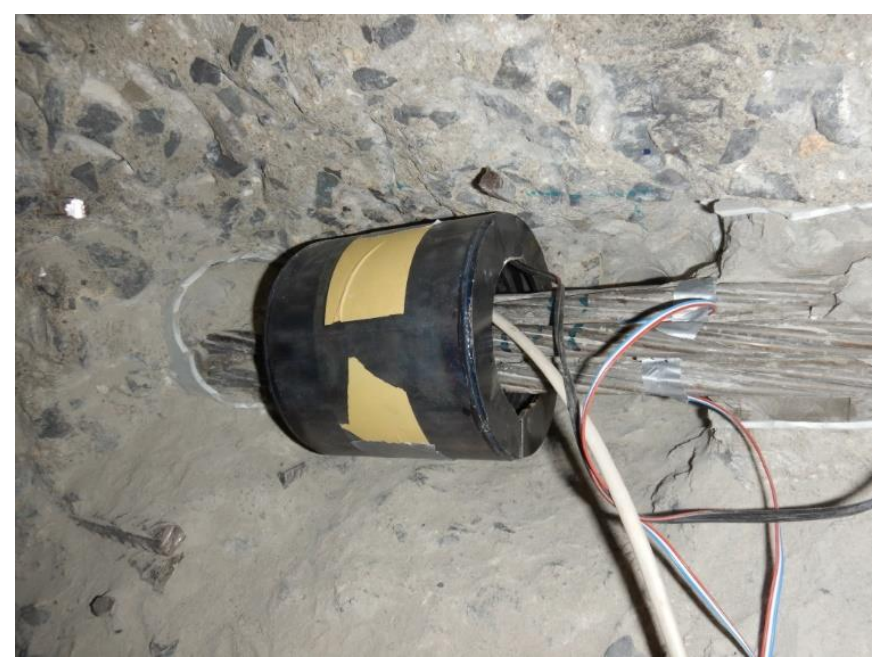

Figure 8 . The post installed ME sensor wounded on the prestressed cable composed of twelve strands Lp $15.7 \mathrm{~mm}$ 


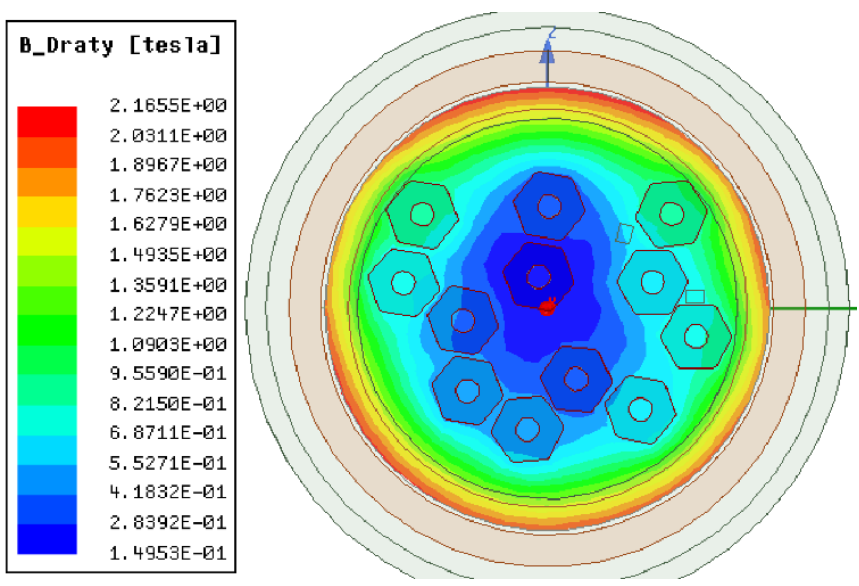

Figure 9. The important output of the numerical model necessary for the correct interpretation of experimental data (the numerical model of the observed cable cross section with precisely located positions of twelve strands Lp15,7 and two used Hall's sensors)

tensioned one and it is composed of twelve strands Lp 15.7. It is led through the web of the box girder made from monolithic concrete inside a thin-wall steel duct and it was injected with cement mortar after its tension. The separates parts of one prestressed cable were interconnected by couplers in working joints.

In the course of the experiment, six fully equipped ME sensors were installed additionally on selected existing prestressed cables. It means, all sensor constructions among others included two Hall's sensors and both secondary coil No. 1 and No. 2.

In general, individual observed cross sections of the investigated prestressed cables are unique at least due to the variable arrangement of the strands in the prestressed cable (see Figure 9 for example), therefore the developed methodology for application of the modified magnetoelastic method on existing prestressed concrete structures that is described in more detail in reference [9] had to be used during the results evaluations. In brief, the prestressed force evaluation in the cable by the methodology consists of four main stages. The first one is the as accurate as possible measurement of the real geometric arrangement of the strands in the observed cross-section of the prestressed cable. The second stage is theoretical modelling of the measured geometric shape of the cable in a 3D software for the electromagnetic field analysis (see Figure 9). The third one is the in situ observation of the electromagnetic behaviour of the ME sensor installed on the cable (see Figure 8). The fourth stage is the application of the available calibration curve that was gained in laboratory test realized for the strand type from which the analysed cable is assembled.

The short study of the result uncertainties caused by the partially variable mutual position of the sensor body and the cable was carried out during the described experiment. The prestressed forces in the observed cables were determined repeatedly three times by the modified magnetoelastic method and they were subsequently mutually compared. For example, the mutual geometric arrangements of the ME sensors and the investigated prestressed cables were changed repeatedly. Each individual ME sensor was partially displaced several times in the plane of observed cable cross section in different directions perpendicular to the longitudinal axis of the cable within a range of clearance gap between the sensor body and the cable surface. Subsequently, the measurement of the prestressed force was realized for the each adjusted mutual position of the sensor body and the cable.

The above-mentioned analysis of the obtained results provided information about partial uncertainties of the evaluated tension forces which are related with the geometric arrangement between the sensor body and the cable and the numerical modelling of the problem (see Figure 9). The variance of the resulting values on each observed cable did not exceed $1 \%$ of the prestressed force magnitudes.

The tensile forces evaluated by the modified magnetoelastic method in the individual observed cable locations were mutually compared as well. The force magnitude of some investigated cables was only about $50 \%$ of the prestressed forces evaluated in other ones. These cables were examined in detail subsequently. Their significant weakening by corrosion was detected near their measured cross section and the corrosion process was distinctly uneven in these cases.

The authors tried to reduce uncertainties of the experiments as much as possible. The main sources of the uncertainties are follows: the uncertainty related to the material of the investigated prestressed reinforcement, the uncertainty connected with the FEM numerical modelling, the uncertainty caused by the actual temperature of the reinforcement in experiment time and the uncertainties of the Hall's sensors.

The chemical compositions and the microscopic structures of the materials of the prestressed reinforcements are more or less different. If it is possible, the reference sample of the investigated prestressed reinforcement should be taken from the observed existing structure and then analysed in an ideal variant of the experiment.

The application of more Hall's sensors to different positions in the investigated cross-section of the prestressed cable should be used for more accurate verification of the numerical model based on the multiple comparison of the experimental and theoretical results.

The evaluating curves (see Figure 7 for example) for particular material of some prestressed reinforcement that characterize the temperature effect on the analysed results should be realized using calibrated force and temperature sensors and devices.

The sensitivity of Hall's sensors applied by the experiments should be verified using a calibrated source of a reference magnetic field.

As can be seen from Figure 8 the installation of the ME sensors was associated with the laborious and precise local demolition of the concrete layer in locations with cables selected for the experiment. The additional benefit of this experiment is fact, that the created openings for the ME sensor installation can be used for a proper inspection of the prestressed reinforcements and their potential corrosion.

Due to the fact that the original post-injection grouting of the steel ducts with the cables was not performed well, the ME sensor can also identify weakening of the cable cross section by corrosion that is located out of the investigated place and the created opening, because there is not redistribution of the prestress losses of the cable tensile stress on other strands or cables.

\subsection{The experiments realized on the prestressed bars}

The experiments described in this chapter were carried out on some samples of the currently used prestressed threadbar of type MUKUSOL 15FS made by Dywidag company. The bar diameter is $15 \mathrm{~mm}$, the thread diameter is $17 \mathrm{~mm}$, its characteristics 
strength is $960 \mathrm{MPa}$ and appropriate characteristics breaking load is $170 \mathrm{kN}$.

In the past years, the authors realized similar experiments focused on the threadbars [11]. However, the simpler constructions of the ME sensors with only one secondary coil were used during these experiments. It was stated in conclusion of the paper [11] that the supervision of the presstressed bars using the modified magnetoelastic method does not seem appropriate.

The supposed advantages of the newly used ME sensor arrangement with both secondary coils were verified on the threadbars in the course of the recent experiments [8]. The secondary coils were applied as the detector of the of magnetic field intensity " $\mathrm{H}$ " instead of the originally used Hall's sensors, that failed in the conditions of the swirling magnetic field caused by the threads of the prestressed bar [11].

The differential signal evaluated from the data observed by the pair of the secondary coils is used for evaluating of the same physical quantity that is also measured by Hall's sensors. However, quantity magnetic field intensity " $\mathrm{H}$ " is not observed only locally as in the case of Hall's sensors but the differential signal corresponds to value of quantity " $\mathrm{H}$ " averaged over the volume of the annulus between two secondary coils. Both secondary coils used in the applied ME sensor were made with the same length as the screw thread pitch of the investigated threadbar for the purpose to reduce the negative influence of the threads and to eliminate all abnormalities of the magnetic field in their neighborhood. See reference [8] for more details.

The phenomenon of the inverse sensitivity of the threadbar material to the applied axial load was found out during the analysis of experimental data. This phenomenon is probably caused by the disturbing effects of eddy currents. The development of their influence is produced by the compact massive cross section of the bar in comparison with prestressed wires or strands. In terms of the magnetic behaviour, the strand is close to a system of prestressed wires and not to the compact threadbar. More details of the phenomenon are described in reference [8].

The maximal difference between the force actual values, that were measured by the standard force sensor used during the experiment, and the force determined using the evaluation curve applied to the experimental data measured by the ME sensor was

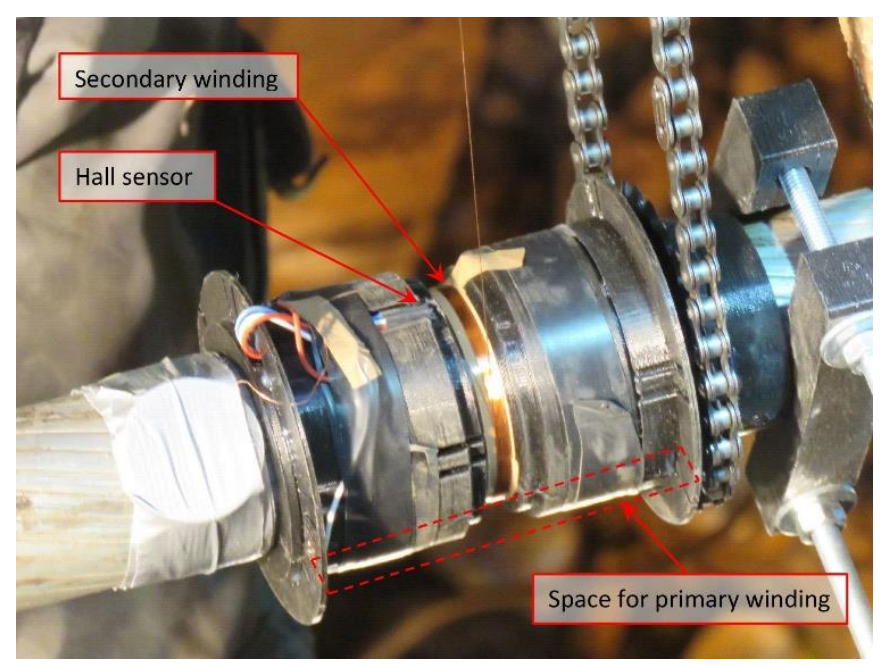

Figure 10. The production of the ME sensor on the cable PV 150, the process of the winding of the secondary coil 1, the placement of Hall's sensor about $2.0 \%$ of the characteristic strength of the investigated threadbar.

Based on the new experiences of the authors with application of the ME sensor containing both secondary coils, it can be stated that modified ME method enables to determine the actual value in the standard prestressed threadbars. However, during the design of an experiment, it is necessary to consider the lower sensitivity of the modified magnetoelastic method for the prestressed threadbars compared to the strands for example. This lower sensitivity is probably related to the lower strength of the material used for production of the threadbars.

\subsection{The experiment realized on the standard full locked cable PV-150}

The experiment described in this part was realized on the currently used standard full locked cable PV 150 made by firm Pfeifer Seil- und Hebetechnik GmbH. The arrangement of the experiment and its results are described in more detail in the reference [8].

The basic objective of the experiment was to verify if the modified ME method can be used for determination of the axial tensile forces in the standardly produced full locked cables of type PV. The core of this cable type is composed of several layers of circular cross-section wires and the locked outer layers are formed from $\mathrm{Z}$ shaped wires. The outer diameter of the investigated cable PV 150 is $40 \mathrm{~mm}$ and its characteristics strength is $1435 \mathrm{MPa}$ and appropriate characteristics breaking load is $1520 \mathrm{kN}$.

The magnetic behaviour of the investigated cable PV 150 was observed by the fully equipped ME sensor (see Figure 1). Its construction involved the following parts: The sensor body made by a 3D printer (see Figure 10), the primary coil used as the controlled magnetic field source, the secondary coil 1 (see Figure 10) applied as a sensor of magnetic flux that is closely related to the magnetic induction " $\mathrm{B}$ " in the measured cable cross section, the system of two Hall sensors (see Figure 10) and the secondary coil 2 used as two independent sensors of magnetic field intensity "H" in a measured cable cross section and steel shield of the ME sensor applied as a sensor protection against magnetic influences from its surroundings.

In the course of the experiment, the cable was anchored in a stand and it was loaded twice in nine force steps by the tensile force produced by a calibrated tensional hydraulic jack.

The experiment was primarily focused on the verification of usability of the modified magnetoelastic method for this type of the structural element and it was not intended as the full experimental analysis of the evaluating curves because it was realized only for one temperature of the cable. However, several various evaluation procedures were examined based on the measured data.

The obtained experimental results show that the modified magnetoelastic method can be applied meaningfully for experimental determination of the tensile force in the standard fully locked cables of type PV. The magnetic behaviour of these cables is analogical to the standard prestressed strands of type Lp.

\section{ALTERNATIVE ARRANGEMENT OF THE ME SENSOR}

In the course of the research project, the design of the prototype of the removable ME sensor was also developed. Its each part can be simply mounted on the observed structural element and then removed easily and no one coil has to be 


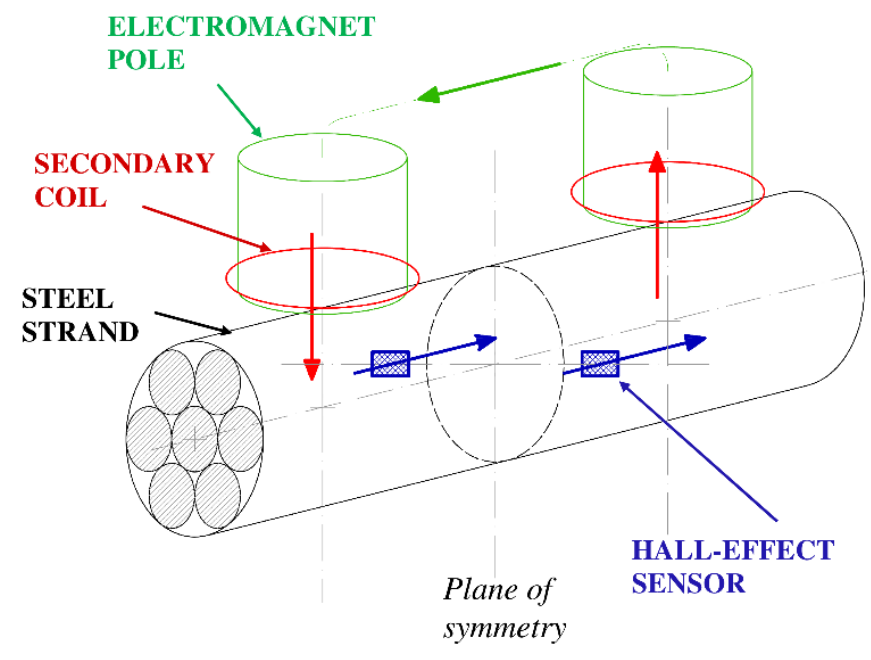

Figure 11. The arrangement of the removable ME sensor

produced in situ, so the time-consuming winding of the coils is not required on the experiment location.

The basic part of the removable ME sensor is the primary coil winded on the steel horseshoe-shaped core (see Figure 12), that is used for developing sufficiently intense variable electromagnetic field. The parameters of the magnetic field are measured applying two secondary coils and two Hall's sensors.

The secondary coils are winded around the orange frames that were made by a 3D printer and that are put on the both ends of the steel core (see Figure 12). They measure the magnetic field properties in a direction perpendicular to the element axis. More specifically, they observed the changes of magnetic-inductive flow in the magnetic circuit, that correlate with the intensity of the magnetic induction " $\mathrm{B}$ ".

Two Hall's sensors are located inside the special black holder (see Figure 12) that was also made by the $3 \mathrm{D}$ printer and that is mounted on the studied structural element in the plane of symmetry of the removable ME sensor. It means, both Hall's sensors are situated in the central plane of the sensor perpendicular to the investigated element and they measure the properties of the magnetic field in a direction parallel with the element axis, more specifically, they observe magnetic field intensity " $\mathrm{H}$ " in the investigated cross section of the element that is located in the plane of sensor symmetry.

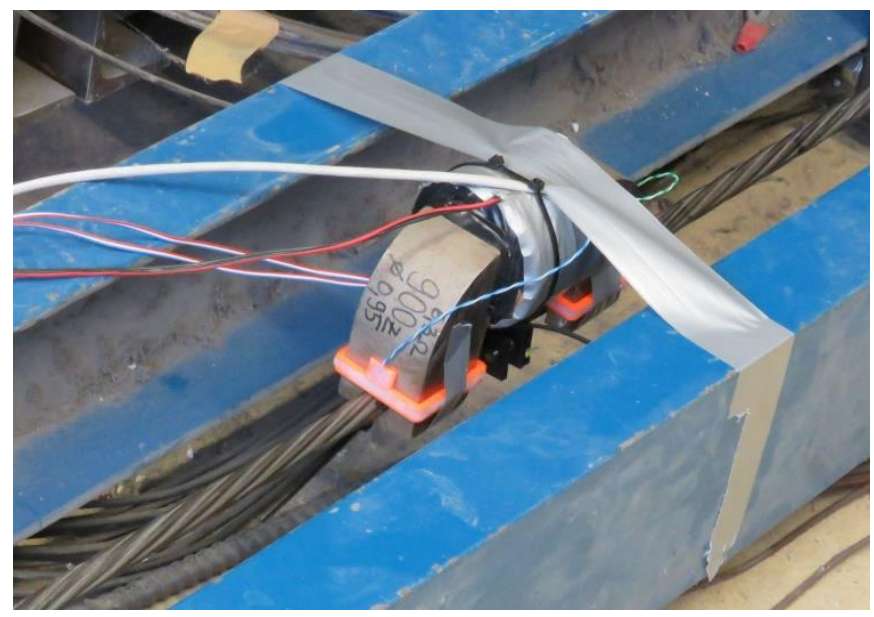

Figure 12. The application of the removable ME sensor on the strand Lp 15.7 in the laboratory conditions
As part of the development of the sensor prototype, the possibility of magnetic-inductive flow observation was evaluated. Flow observations were made by using two measuring coils that are positioned on the both ends of the steel horseshoe-shaped core of the electromagnet and that are connected in series in the measuring circuit.

The mutual relation between the time courses of the measured signals from the secondary coils and Hall's sensors was investigated and assessed.

The subject of interest was the answer for the question if the magnetization of the compact massive core of the primary coil, on which both secondary coils are threaded, does not influence the measurement of hysteresis behaviour of the investigated prestressed element.

Based on the obtained experimental data, it can be stated that the hysteresis is affected by the compact core of the primary coil. However, this phenomenon occurs only during the rapid increase of magnetic flux during the rapid increase of the excitation electric pulse. It always disappears completely during the descending branch of the excitation pulse because then the phenomenon is almost quasi-static from the view of the dynamics of the magnetic field development.

The magnetic behaviour of the assessed arrangement of the experiment, it means the system consisting of the removable ME sensor and the investigated structural element, is purely linear in the zone of the descending branch of the excitation pulse that is really used for the analysis of the experimental data measured by the ME sensor. This behaviour was also verified by the removable ME sensor, which was mounted on a nonferromagnetic dummy of the investigated element.

The obtained results of the experiment, that are described in more detail in reference [7], confirmed the functionality of the designed arrangement of the removable ME sensor. However, the precision of the gained results is lower than for the cylindrical ME sensor, that is described in the chapter 3, due to the less accurate definition of the magnetic field in the observed cross section compared to the arrangement of the cylindrical ME sensor.

The removable ME sensor can be used for a relatively quick preparative experimental analysis intended for the evaluation of the total value of the prestressed force in the structural elements activated before the start of the experiment. The intended purpose of the practical application of the removable ME sensor is a quick selection of prestressed elements that are the most important for further detailed analysis by using the cylindrical fully equipped ME sensors.

The comparison of the basic advantages and disadvantages between the standard cylindrical fully equipped ME sensor whose general scheme is shown in Figure 1 and the removable ME sensor whose general diagram is drawn in Figure 11 could be summarized in following notes.

The advantages of the standard cylindrical ME sensor are that a relatively high measurement accuracy could be achieved and that the dimension of the cross-section of the observed prestressed reinforcement is not restricted practically. Its basic disadvantage is its relatively time-consuming production.

The fundamental advantage of the removable ME sensor is that the time necessary for preparation and realization of the experiment is substantially shorter. Its disadvantages are higher uncertainties of the evaluated prestressed forces and a considerable limitation of the dimension of the observed reinforcement cross-section. 


\section{RESULTS AND DISCUSSIONS}

So far, as part of development of the modified magnetoelastic method, the experiments focused on the evaluating curves were realized for four specimens of the patent wires, three types of the prestressed bars and two prestressed strands Lp 15.7 / $1860 \mathrm{MPa}$ from different producers.

The results obtained for six selected investigated prestressed elements (namely, three patent wires P4.5, one patent wire P7 and two prestressed strands Lp15.7) were compared mutually in detail. The resultant regression fitting curves for the particular elements at the element temperature $20^{\circ} \mathrm{C}$ are drawn in Figure 7.

The analysis of the results shows that the standard deviations of all measured data from the specific calculated evaluating curves are usually significantly lower than $1.0 \%$ of the design strength.

The comparison of the corresponding evaluating curves for two different samples of the prestressed strands Lp 15.7 / 1860 $\mathrm{MPa}$ made by different producers is shown in Figure 7 (specimens "L 2019" and "L 2016"). A specific producer of the strand "L 2016" is not known. The strand "L 2019" was supplied by Freyssinet CZ. The difference between curves is roughly about $5 \%$ of the design strength of the strands. It means the evaluating curves of the particular samples of the strand Lp 15.7 deviate roughly about $2.5 \%$ from the averaging evaluating curve of this type of the prestressed strand.

Very similar results were obtained from the comparison of the corresponding evaluating curves for three different test samples of the patent wire P4.5 that were taken during the demolitions of three different existing bridges. These bridges were built in the seventies and eighties in Czechoslovakia. For example, three curves "D4 spec. 1", "D4 spec. 2" and "D4 spec. 3" for the wire temperature $20{ }^{\circ} \mathrm{C}$ are drawn in Figure 7 . The evaluating curves of the particular test samples of the patent wire P4.5 deviate roughly about $2.5 \%$ from the averaging evaluating curve of this type of patent wire.

In contrast to the previously mentioned results, the significant difference roughly above $20 \%$ of the design strength was found between the evaluating curves for the patent wires P7 and P4.5, as can be seen from Figure 7.

Results of the additional analyses indicated that the chemical compositions of the materials, from which the studied wires P4.5 and $\mathrm{P} 7$ were made, were almost identical. Nevertheless, for the wire P7, it was found out, that its microscopic structure of steel is fundamentally different from the three wires P4.5 and this fact seems to be the reason of the difference between the evaluating curves for the patent wire P7 and wires P4.5.

As it was expected, it was confirmed experimentally that the evaluation relationships are not applicable universally for different materials used for various prestressed elements. It was further found that the evaluation curves are not mutually applicable even for the patent wires with different diameters.

\section{CONCLUSIONS AND OUTLOOK}

The results stated above demonstrate that the modified magnetoelastic method can be used for the experiments realized on the existing structures for the determination of the actual value of the tension force in steel prestressed structural elements using the available general evaluating curves. The result uncertainties of these experiments are then similar as for the alternative experimental methods, e.g., the vibration frequency method [1], [2]. It should be noted here that the frequency method cannot be used for the prestressed elements embedded in the concrete.

In the cases when it is possible to remove the test sample of the specific prestressed element from the particular existing bridge then the evaluating curves for this observed element can be evaluated according to the above-described procedure. The uncertainties of the evaluated prestressed forces are then relatively small and they are comparable with precision of the method based on a strain measurement with strain gauges.

The possibility of using the modified magnetoelastic method for the prestressed bars was also verified during previous experiments [8] and [11]. Based on the new experiences of the authors with application of the ME sensor containing both secondary coils, it can be stated that modified magnetoelastic method enables to determine the actual value in the standard prestressed threadbars. However, the ME sensor sensitivity, when it is applied on the threadbars, is lower than for the prestressed wires and strands. The main reason of this fact is the significantly lower design strength of the threadbar materials.

According to the authors opinion, the modified magnetoelastic method is the only one that can be purposefully and effectively used for the prestressed force evaluation in the prestressed reinforcements embedded inside the concrete on the existing prestressed concrete bridges or similar engineering structures.

\section{ACKNOWLEDGEMENT}

The results presented in this article are outputs of the research project FV 30457 "Utilization of a Magnetoelastic Method for Increasing the Reliability and Durability of Existing and Newly Built Prestressed Concrete Structures" supported by Ministry of Industry and Trade of the Czech Republic.

\section{REFERENCES}

[1] M. Polák, T. Plachý, Determination of Forces in Roof Cables at Administrative Center Amazon Court, Procedia Engineering, Vol. 48., 2012, pp. 578-582.

DOI: $10.1016 /$ ij.proeng.2012.09.556

[2] M. Polák, T. Plachý, Experimental Evaluation of Tensile Forces in Short Steel Rods, Applied Mechanics and Materials, Vol. 732, 2015, pp. 333-336.

DOI: $10.4028 /$ www.scientific.net\%2FAMM. 732.333

[3] P. Fajman, M. Polák, Measurement of structural cable of membranes, Proceedings of the 50th Annual Conference on Experimental Stress Analysis EAN 2012, Tábor, Czech Republic, 4-7 June 2012, pp. 61-64.

[4] J. Máca, P. Fajman, M. Polák, Measurements of forces in cable membrane structures, Proceedings of the 13th International Conference on Civil, Structural and Environmental Engineering Computing CC 2011, Chania - Crete, Greece, 6-9 September 2011, paper 190. DOI: $10.4203 /$ ccp. 96.190

[5] P. Fajman, M. Polák, J. Máca, T. Plachý, The Experimental Observation of the Prestress Forces in the Structural Elements of a Tension Fabric Structure, Applied Mechanics and Materials, Vol. 486, 2014, pp. 189-194.

DOI: $10.4028 /$ www.scientific.net/AMM.486.189

[6] T. Klier, T. Míčka, M. Polák, M. Hedbávný, Application of the Modified Magnetoelastic Method, Proceedings of the 17th IMEKO TC 10 and EUROLAB Virtual Conference "Global Trends in Testing, Diagnostics \& Inspection for 2030", Online event, 20-22 October 2020, pp. 344-349. Online [Accessed 8 September 2021] https://www.imeko.org/publications/tc10-2020/IMEKOTC10-2020-051.pdf 
[7] T. Klier, T. Míčka, M. Polák, T. Plachý, M. Hedbávný, L. Krejčíková, The modified elastomagnetic sensor intended for a quick application on an existing prestressed concrete structures, Proceedings of the 58th Conference on Experimental Stress Analysis EAN, Sobotín, 19-22 October 2020, p. 9.

[8] T. Klier, T. Míčka, M. Polák, T. Plachý, M. Hedbávný, L. Krejč́ková, New Information about Practical Application of the Modified Magnetoelastic Method, MATEC Web of Conferences, Vol. 310, No. 00026, 2020, p. 10.

DOI: $10.1051 /$ matecconf/202031000026

[9] T. Klier, T. Míčka, M. Polák, T. Plachý, M. Hedbávný, R. Jelínek, F. Bláha, Application of the Modified Magnetoelastic Method and an Analysis of the Magnetic Field, Acta Polytechnica CTU Proceedings, Vol. 15., 2018, pp. 46-50. DOI: $10.14311 /$ APP.2018.15.0046

[10] T. Klier, T. Míčka, T. Plachý, M. Polák, T. Smeták, M. Šimler, The verification of a new approach to the experimental estimation of tensile forces in prestressed structural elements by method based on the magnetoelastic principle, MATEC Web of Conferences, Vol. 107, No. 00015, 2017, p. 8 DOI: $10.1051 /$ matecconf/201710700015

[11] T. Klier, T. Míčka, M. Polák, T. Plachý, M. Šimler, T. Smeták, The in Situ Application of a New Approach to Experimental Estimation of Tensile Forces in Prestressed Structural Elements by Method Based on the Magnetoelastic Principle, Proceedings of the 55th Conference on Experimental Stress Analysis 2017, Košice, Czechia, 30 May - 1 June 2017, pp. 122-132.

[12] P. Sumitro, N. Miyamoto, A. Jaroševič, Corrosion monitoring by utilizing EM technology, Proceedings of the 5th International Conference on Structural Health Monitoring of Intelligent Infrastructure, SHMII-5 2011, Cancun, Mexico, 11-15 December 2011, p. 11.

[13] M. Chandoga, A. Jaroševič, J. Sedlák, E. Sedlák, Experimental and in situ study of bridge beams supported by bottom external tendons, Proceedings of the 3rd International fib Congress and Exhibition, Incorporating the PCI Annual Convention and Bridge Conference 2010, Washington, USA, 29 May - 2 June 2010, 9 pp.

[14] M. Chandoga, A. Jaroševič, Measurement of force distribution along the external tendons, Proceedings of the International Conference Analytical Models and New Concepts in Concrete and Masonry Structures, Lodz, Poland, 9-11 June 2008, 6 pp.

[15] M. Chandoga, P. Fabo, A. Jaroševič, Measurement of Forces in the Cable Stays of the Apollo Bridge, Proceedings of the $2 \mathrm{nd} \mathrm{fib}$ Congress, Naples, Italy, 2006, pp. 674-675.

[16] P. Fabo, M. Chandoga, A. Jarosevic, The smart tendons - A new approach to prestressing, Proceedings of the fib Symposium 2004
- Concrete Structures: The Challenge of Creativity, Avignon, France, 26-28 April 2004, pp. 286-287.

[17] P. Fabo, A. Jaroševič, M. Chandoga, Health monitoring of the steel cables using the elasto-magnetic method, Proceedings of the ASME International Mechanical Engineering Congress and Exposition, 2002, pp. 295-299. DOI: 10.1115 /IMECE2002-33943

[18] S. Sumitro, A. Jaroševič, M. L. Wang, Elasto-magnetic sensor utilization on steel cable stress measurement, Proceedings of the 1st Fib Congress, Concrete Structures in the 21th Century, 2002, pp. 79-86.

[19] M. Chandoga, J. Halvonik, A. Jaroševič, D. W. Begg, Relationship between design, modelling and in-situ measurement of pretensioned bridge beams, Proceedings of the 8th International Conference on Computational Methods and Experimental Measurements, CMEM 1997, Rhodes, Greece, 1 May 1997, pp. 623-632.

[20] A. Jaroševič, Magnetoelastic Method of Stress Measurement in Steel, NATO Science Series, 3 (65), 1998, pp. 107-114.

[21] A. Jaroševič, M. Chandoga, Force Measuring of Prestressing Steel, Inzinierske Stavby, 42, 1994, pp. 56-62.

[22] A. M. Sarmento, A. Lage, E. Caetano, J. Figueiras, Stress measurement and material defect detection in steel strands by magneto elastic effect. Comparison with other non-destructive measurement techniques, Proceedings of the 6th International Conference on Bridge Maintenance, Safety and Management IABMAS 2012, Stresa-Lake Maggiore, Italy, 8-12 July 2012, pp. 914-921. DOI: $10.1201 / \mathrm{b} 12352-126$

[23] C. Chen, W. Wu, D. Wei, Stress measurement of pre-stressed members using elasto-magnetic sensors, Journal of the Chinese Institute of Civil and Hydraulic Engineering, Vol. 24, Iss. 2, 2012, pp. 157-167.

[24] H. J. Wichmann, A. Holst, H. Budelmann, Magnetoelastic stress measurement and material defect detection in prestressed tendons using coil sensors, Proceedings of 7 th International Symposium on Non-Destructive Testing in Civil Engineering NDTCE'09, Nantes, France, 30 June - 3 July 2009, 6 pp. Online [Accessed 8 September 2021] https://www.ndt.net/article/ndtce2009/papers/60.pdf

[25] H. Feng, X. Liu, B. Wu, D. Wu, X. Zhang, C. He, Temperatureinsensitive cable tension monitoring during the construction of a cable-stayed bridge with a custom-developed pulse elastomagnetic instrument, Structural Health Monitoring, Vol. 18, Iss. 5-6, 2019, pp. 1982-1994. DOI: $\underline{10.1177 / 1475921718814733}$ 